\section{Correspondance on 'Clinical characteristics and genetic analyses of 187 patients with undefined autoinflammatory diseases'}

We read with great interest the article by Ter Haar $e t a l^{1}$ who describe the clinical characteristics, treatment response and genetic findings in a cohort of patients $(n=187)$ with undefined systemic autoinflammatory diseases (sAIDs). The authors observed that patients with pericarditis were older at disease onset and had milder disease whereas patients with intellectual impairment were younger at disease onset and often had affected relatives. Of note, among patients with recurrent disease course, $30 \%(\mathrm{n}=53 / 180)$ had aphthous stomatitis. Moreover, after complete gene screening, screening of most relevant exons or screening of most relevant point mutations, 9.4\% ( $\mathrm{n}=15 / 159)$ of patients carried likely pathogenic variants or variants of uncertain significance. Our recent French multicentre study on targeted next-generation sequencing (NGS) gives further insight into the clinical characteristics and genetic findings in patients with sAIDs associated with recurrent aphthous stomatitis (RAS).

RAS (ie, adult onset, association with other features such as fever and malaise) can represent a diagnostic challenge. RAS especially when associated with fever, pharyngitis and lymphadenopathy may be the first presentation of sAIDs. ${ }^{2}$ sAIDs arise from impaired inflammatory responses due to variants in gene coding proteins involved in innate immunity. commonly of monogenic inheritance. ${ }^{3}$ The most common sAIDs associated with RAS are periodic fever, aphthous stomatitis, pharyngitis and adenopathy syndrome, NLRP3-associated autoinflammatory disease (NLRP3-AID), tumour necrosis factor receptorassociated periodic syndrome (TRAPS), A20 haploinsufficiency (HA20), mevalonate kinase deficiency (MKD) and deficiency of adenosine deaminase 2 (DADA2). ${ }^{4}$ NGS allows for investigating multiple genes simultaneously and has become the preferred method for molecular testing of sAIDs. ${ }^{5}$

In our study, we aimed to determine the diagnostic performance of a targeted NGS panel of 55 genes in patients with suspected sAID with RAS.

A total of 631 adult and paediatric patients were referred for genetic diagnosis of sAID to the reference laboratory for autoinflammatory diseases of Montpellier, France, between 2014 and 2019. Patients of whom clinical information was available $((\mathrm{n}=338), 53.3 \%$ female, median age 15 years, $107(31.7 \%)$ with RAS) were included in the final analysis (table 1 ).

We detected pathogenic or likely pathogenic variants compatible with a final diagnosis in 41/338 (12.1\%) of patients. These variants were found in the following genes: $M E F V(\mathrm{n}=7), M V K$ $(\mathrm{n}=6)$, NLRP3 $(\mathrm{n}=4)$, TNFRSF1A $(\mathrm{n}=4)$, ADA2 $(\mathrm{n}=3)$, NOD2 $(\mathrm{n}=3), \operatorname{NLRC4}(\mathrm{n}=2), \operatorname{SLC} 29 A 3(\mathrm{n}=2)$, TMEM173 $(\mathrm{n}=2)$, others $(n=8)$ (table 1). Based on the results of the genetic screening, probable/confirmed diagnosis were: familial Mediterranean fever, $(\mathrm{n}=5)$, MKD $(\mathrm{n}=6)$, NLRP3-AID $(\mathrm{n}=4)$, TRAPS $(\mathrm{n}=4)$, DADA2 $(\mathrm{n}=3)$, Blau syndrome $(\mathrm{n}=3)$, NLRC4-associated autoinflammatory disease (NLRC4-AID) $(\mathrm{n}=2), \mathrm{H}$ syndrome $(n=2)$, STING-associated vasculopathy with onset in infancy $(n=2)$, pyrin-associated autoinflammation with neutrophilic dermatosis (PAAND) $(n=2)$, HA20 $(n=1)$, others $(n=7)$.

Pathogenic or likely pathogenic variants were detected in 9/107 $(8.4 \%)$ of patients with RAS: MVK $(\mathrm{n}=3)$, TNFRSF1A $(\mathrm{n}=2)$, $\operatorname{MEFV}(\mathrm{n}=1), A D A 2(\mathrm{n}=1), \operatorname{NLRP3}(\mathrm{n}=1)$, TRNT1 $(\mathrm{n}=1)$. Probable/confirmed diagnosis of these patients were: $\operatorname{MKD}(n=3)$, TRAPS $(\mathrm{n}=2)$, PAAND $(\mathrm{n}=1)$, DADA2 $(\mathrm{n}=1)$, NLRP3-AID
Table 1 Demographic and clinical characteristics of patients with suspected systemic autoinflammatory disease (sAIDs) and genotypes of the 41 patients with a probable or confirmed diagnosis of monogenic SAIDs using a targeted next-generation sequencing panel

\begin{tabular}{|c|c|c|c|c|}
\hline Variable & $\begin{array}{l}\text { Total } \\
(n=338)\end{array}$ & $\begin{array}{l}\text { No RAS } \\
(n=231)\end{array}$ & $\begin{array}{l}\text { RAS } \\
(n=107)\end{array}$ & $P$ value \\
\hline $\begin{array}{l}\text { Age (years), } \\
\text { median (IQR) }\end{array}$ & $15(8-32)$ & $15(8-32)$ & $\begin{array}{l}16 \\
(11-30)\end{array}$ & 0.54 \\
\hline Females, n (\%) & $182(53.3)$ & $124(53.7)$ & $58(54.2)$ & 1 \\
\hline $\begin{array}{l}\text { Consanguinity, } \\
\mathrm{n}(\%)\end{array}$ & $8(2.4)$ & $6(2.6)$ & $2(1.9)$ & 1 \\
\hline $\begin{array}{l}\text { Frequency of } \\
\text { attacks }>1 / \\
\text { month, } n(\%)\end{array}$ & $189(55.9)$ & $100(50)$ & $49(53)$ & 0.35 \\
\hline Fever, n (\%) & $265(78.4)$ & $185(76)$ & $92(82)$ & 0.40 \\
\hline Arthritis, n (\%) & 99 (29.3) & $75(31)$ & $31(27)$ & 0.44 \\
\hline $\begin{array}{l}\text { Genital ulcers, } \\
\text { n (\%) }\end{array}$ & $18(5.3)$ & $0(0.0)$ & $18(16.8)$ & $<0.0001$ \\
\hline $\begin{array}{l}\text { Pleuritis/ } \\
\text { pericarditis, } \\
\text { n (\%) }\end{array}$ & 41 (12.1) & 34 (14.7) & $7(6.5)$ & 0.032 \\
\hline $\begin{array}{l}\text { Diarrhoea, } \\
n(\%)\end{array}$ & 95 (28.1) & $52(22.5)$ & $43(40.2)$ & 0.0001 \\
\hline $\begin{array}{l}\text { Erythema } \\
\text { nodosum, } \\
\text { n (\%) }\end{array}$ & $19(5.6)$ & $11(4.8)$ & $8(7.5)$ & 0.32 \\
\hline Uveitis, n (\%) & $24(7.1)$ & $12(5.2)$ & $12(11.2)$ & 0.066 \\
\hline $\begin{array}{l}\text { Meningitis, } \\
\mathrm{n}(\%)\end{array}$ & $21(6.2)$ & $10(4.3)$ & $11(10.3)$ & 0.05 \\
\hline Vasculitis, n (\%) & $30(8.9)$ & $18(7.8)$ & $12(11.2)$ & 0.31 \\
\hline $\begin{array}{l}\text { Cytopenia, } \\
\text { n (\%) }\end{array}$ & $19(5.6)$ & $16(6.9)$ & $3(2.8)$ & 0.20 \\
\hline $\begin{array}{l}\text { Immune } \\
\text { deficiency, } \\
\mathrm{n}(\%)\end{array}$ & $36(10.7)$ & $21(9.1)$ & $15(14.0)$ & 0.19 \\
\hline $\begin{array}{l}\text { Genetic } \\
\text { diagnoses, n } \\
\text { (\%): }\end{array}$ & $41(12.1)$ & $32(13.9)$ & $9(8.4)$ & 0.21 \\
\hline
\end{tabular}

$(\%)$

\begin{tabular}{|c|c|}
\hline Blau syndrome & NOD2: p.(Lys225Met);(Lys225=) \\
\hline $\begin{array}{l}\text { Blau } \\
\text { syndrome }\end{array}$ & NOD2: p.(Arg334GIn);(Arg334=) \\
\hline Blau syndrome & NOD2: p.(Cys483Arg);(Cys483=) \\
\hline DADA2 & ADA2: p.(Cys159Tyr);(Tyr453Cys) \\
\hline DADA2 & ADA2: p.(Gly47Arg);(Gly47Arg) \\
\hline DADA2* & ADA2: p.(Ile143Serfs*41);(?) \\
\hline DITRA & IL36RN: p.(His32Arg);(His32Arg) \\
\hline FMF & MEFV: p.(Met694lle);(Met694=) \\
\hline FMF & MEFV: p.(Met694Val);(Met694=) \\
\hline FMF & MEFV: p.(Met694Val);(Met694=) \\
\hline FMF & MEFV: p.(Met694Val);(Met694=) \\
\hline FMF & MEFV: p.(Met694Val);(Met694=) \\
\hline H syndrome & SLC29A3: p.(?);(?) \\
\hline H syndrome & SLC29A3: p.Gly427Ser(;)Gly427Ser \\
\hline HA20 & TNFAIP3: p.(Pro274Hisfs*13);(Pro274=) \\
\hline JIA & $\begin{array}{l}\text { LACC1: } \\
\text { p.(Asp125Metfs*12);(Asp125Metfs*12) }\end{array}$ \\
\hline $\begin{array}{l}\text { Majeed } \\
\text { syndrome }\end{array}$ & LPIN2: p.Gln44Hisfs*13(;) Gln44Hisfs*13 \\
\hline MKD & MVK: p.(Ile268Thr);(Val377Ile) \\
\hline MKD & MVK: p.(Lys13Gln);(Val377lle) \\
\hline MKD* & MVK: p.(Thr237Ser);(Val377Ile) \\
\hline MKD* & MVK: p.(Thr237Ser);(Val377lle) \\
\hline MKD & MVK: p.(Ile268Thr);(Val377lle) \\
\hline MKD* & MVK: p.(Thr342Ile);(Val377Ile) \\
\hline NLRC4-AID & NLRC4: p.(Thr177Ser);(Thr177=) \\
\hline NLRC4-AID & NLRC4: $\mathrm{p}$.(Thr337Asn);(Thr337=) \\
\hline NLRP12-AID & NLRP12: p.(?);(=) \\
\hline NLRP3-AID* & NLRP3: p.(Tyr570=/Asn) \\
\hline
\end{tabular}




\begin{tabular}{|c|c|c|c|c|}
\hline Variable & $\begin{array}{l}\text { Total } \\
(\mathrm{n}=338)\end{array}$ & $\begin{array}{l}\text { No RAS } \\
(\mathrm{n}=231)\end{array}$ & $\begin{array}{l}\text { RAS } \\
(n=107)\end{array}$ & $P$ value \\
\hline NLRP3-AID & NLRP3: p.(Arg260Pro);(Arg260=) & & & \\
\hline NLRP3-AID & NLRP3: p.(Ser726Gly);(Ser726=) & & & \\
\hline NLRP3-AID & NLRP3: p.(Glu688Lys);(Glu688=) & & & \\
\hline PAAND* & MEFV: p.(Ser242Arg);(Ser242=) & & & \\
\hline PAAND* & MEFV: p.(Ser242Arg);(Ser242=) & & & \\
\hline PAPA & PSTPIP1: p.(Glu250Gln);(Glu250=) & & & \\
\hline PRAAS & PSMB8: p.(Thr75Met);(Thr75Met) & & & \\
\hline SAVI & TMEM173: p.(Val155Met);(Val155=) & & & \\
\hline SAVI & TMEM173: p.(Val155Met);(Val155=) & & & \\
\hline SIFD* & TRNT1: p.(His391Arg);(His391Arg) & & & \\
\hline TRAPS* & TNFRSF1A: p.(Cys72Tyr);(Cys72=) & & & \\
\hline TRAPS & TNFRSF1A: p.(Gly204Cys);(Gly204=) & & & \\
\hline TRAPS & TNFRSF1A: p.(Cys72Tyr);(Cys72=) & & & \\
\hline TRAPS* & TNFRSF1A: p.(Thr79Met);(Thr79=) & & & \\
\hline
\end{tabular}

$P$ values are from $\chi 2$ test or Student's $t$ test

In bold: patients with a confirmed SAID (typical clinical presentation associated with a confirmatory genetic test).

*Patients with recurrent aphthous stomatitis (RAS).

AID, associated autoinflammatory disease; DADA2, deficiency of adenosine deaminase 2 ; DITRA, deficiency of interleukin thirty-six receptor antagonist; FMF, familial Mediterranean fever; HA2O A20 haploinsufficiency; JIA, juvenile idiopathic arthritis; MKD, mevalonate kinase deficiency; PAAND, pyrin-associated autoinflammation with neutrophilic dermatosis; PAPA, pyogenic arthritis-pyoderma gangrenosum-acne syndrome; PRAAS, proteasome-associated autoinflammatory syndrome; SAVI, STING-associated vasculopathy with onset in infancy; SIFD, syndrome of congenital sideroblastic anaemia, B-cell immunodeficiency, periodic fevers, and developmental delay; TRAPS, tumour necrosis factor receptor-associated periodic syndrome .

$(n=1)$, syndrome of congenital sideroblastic anaemia, B-cell immunodeficiency, periodic fevers, and developmental delay (SIFD) $(n=1)$. None of the patients had a diagnosis of HA20. Details about single patients are reported in table 1.

When comparing the characteristics of patients with suspected sAID with and without RAS, the latter was associated with more severe illness with genital ulcers, diarrhoea and uveitis being more frequent in these patients. There were no statistically significant differences between the two groups with respect to age, gender, heredity, consanguinity or other clinical symptoms. Furthermore, the presence of RAS was not associated with better diagnostic performances of NGS panel with 9/107 (8.4\%) and $32 / 231(13.9 \%)$ probable/confirmed diagnosis in patients with and without RAS, respectively $(\mathrm{p}=0.21)$.

Failure to diagnose and delay in diagnosis of sAIDs can result in life-threatening consequences. Having an accurate genetic diagnosis is essential to initiate early treatment. Currently, NGS coupled to clinical information has become the preferred method for diagnosing sAIDs. However, the diagnostic utility of targeted genetic panels is usually low (4\%-22\% diagnostic yield). ${ }^{67}$ Since RAS is a frequent manifestation of sAIDs, we hypothesised that its presence could increase the genetic diagnosis yield of NGS. When focusing on patients with RAS, the diagnostic yield did not increase compared with patients without RAS, thus suggesting that this is not a good clinical feature to focus on. Therefore, identifying better clinical factors should be a priority to guide DNA screening.

\section{Gonçalo Boleto $\odot$, ${ }^{1,2,3,4}$ Carolla El Chamieh, ${ }^{5}$ Lucie Biard, ${ }^{5}$ Isabelle Koné-Paut $\odot{ }^{6}{ }^{6}$ Veronique Hentgen, ${ }^{7}$ Guilaine Boursier, ${ }^{8}$ Guillaume Sarrabay, ${ }^{8}$ Patrice Cacoub, ${ }^{1,2,3,4}$ Isabelle Touitou, ${ }^{8}$ David Saadoun ${ }^{1,2,3,4}$}

${ }^{1} \mathrm{AP}$-HP, Groupe Hospitalier Pitié-Salpêtrière, Department of Internal Medicine and Clinical Immunology, Hopitaux Universitaires Pitie Salpetriere-Charles Foix, Paris, France

2INSERM, UMR_S 959, F-75013, Paris, France; CNRS, FRE3632, F-75005, INSERM, Paris, France
${ }^{3}$ UPMC Univ Paris 06, UMR 7211, and Inflammation-Immunopathology-Biotherapy Department (DHU i2B), F-75005, Sorbonne Université, Paris, France

${ }^{4}$ Centre de Référence des Maladies Auto-Immunes et Systémiques Rares, Centre de Référence des Maladies Auto-Inflammatoires et de l'Amylose, Paris, France

${ }^{5}$ Department of Biostatistics and Medical Information, INSERM UMR1153 ECSTRRA Team, Hôpital Saint Louis, AP-HP, Hopital Saint-Louis, Paris, Île-de-France, France ${ }^{6}$ Department of Paediatric Rheumatology, Bicêtre Hospital, APHP, University Paris Sud-Saclay, UVSQ, Kremlin Bicêtre, Hôpital Bicêtre, AP HP, Université Paris Sud,

Bicètre, France

${ }^{7}$ Department of General Pediatrics, CEREMAIA, CH Versailles, Hôpital Mignot, Le Chesnay, France

${ }^{8}$ Cellules souches, plasticité cellulaire, médecine régénératrice et immunothérapies, INSERM, Univ Montpellier, Department of Medical Genetics, Rare Diseases and Personalized Medicine, CEREMAIA, CHU Montpellier, CHRU de Montpellier, Montpellier, France

Correspondence to Dr Gonçalo Boleto, AP-HP, Groupe Hospitalier Pitié-Salpêtrière, Department of Internal Medicine and Clinical Immunology, F-75013, Hopitaux Universitaires Pitie Salpetriere-Charles Foix, Paris, France; goncalo.boleto@aphp.fr

Contributors All authors were involved in drafting the article or revising it critically for important intellectual content, and all authors approved the final version to be submitted for publication. DS had full access to all of the data in the study and takes responsibility for the integrity of the data and the accuracy of the data analysis.

Funding The authors have not declared a specific grant for this research from any funding agency in the public, commercial or not-for-profit sectors.

Competing interests DS has received consulting and lecturing fees from Medimmune, Abbvie, Bristol Meyer Squibb, Celgene, Sanofi, Roche, Servier, Gilead, AstraZeneca and Glaxo Smith Kline. PC has received consulting and lecturing fees from Abbvie, Astra Zeneca, Bristol-Myers Squibb, Gilead, Glaxo Smith Kline, Janssen, Merck Sharp Dohme, Roche, Servier and Vifor. IK-P has received consulting fees from Novimmune, SOBI, Novartis, AbbVie, Roche/CHUGAI, Pfizer, LFB, BMS.

Patient and public involvement Patients and/or the public were not involved in the design, or conduct, or reporting, or dissemination plans of this research.

Patient consent for publication Not required.

Provenance and peer review Not commissioned; internally peer reviewed.

(C) Author(s) (or their employer(s)) 2020. No commercial re-use. See rights and permissions. Published by BMJ.

\section{Check for updates}

To cite Boleto G, El Chamieh C, Biard L, et al. Ann Rheum Dis Epub ahead of print: [please include Day Month Year]. doi:10.1136/annrheumdis-2020-219566

Received 20 November 2020

Accepted 24 November 2020

Ann Rheum Dis 2020;0:1-2. doi:10.1136/annrheumdis-2020-219566

\section{ORCID iDs}

Gonçalo Boleto http://orcid.org/0000-0001-5891-1868

Isabelle Koné-Paut http://orcid.org/0000-0001-8939-5763

\section{REFERENCES}

1 Ter Haar NM, Eijkelboom C, Cantarini L, et al. Clinical characteristics and genetic analyses of 187 patients with undefined autoinflammatory diseases. Ann Rheum Dis 2019;78:1405-11.

2 Pinto A, Lindemeyer RG, Sollecito TP. The PFAPA syndrome in oral medicine: differential diagnosis and treatment. Oral Surg Oral Med Oral Pathol Oral Radiol Endod 2006:102:35-9.

3 Touitou I, Koné-Paut I. Autoinflammatory diseases. Best Pract Res Clin Rheumatol 2008;22:811-29.

4 Scully C, Hodgson T. Recurrent oral ulceration: aphthous-like ulcers in periodic syndromes. Oral Surg Oral Med Oral Pathol Oral Radiol Endod 2008;106:845-52.

5 Rowczenio D, Shinar Y, Ceccherini I, et al. Current practices for the genetic diagnosis of autoinflammatory diseases: results of a European molecular genetics quality network survey. Eur J Hum Genet 2019;27:1502-8.

6 Omoyinmi E, Standing A, Keylock A, et al. Clinical impact of a targeted nextgeneration sequencing gene panel for autoinflammation and vasculitis. PLoS One 2017:12:e0181874.

7 Boursier G, Rittore C, Georgin-Lavialle S, et al. Positive impact of expert reference center validation on performance of next-generation sequencing for genetic diagnosis of autoinflammatory diseases. Journal of Clinical Medicine 1729;2019:8. 